\title{
Comparison of Effects of Low Level Laser and Ultrasound Therapy in Plantar Fasciitis
}

\author{
Aiesha Malik ${ }^{1}$, Saima Riaz ${ }^{2}$, Fatima Mehmood $^{3}$, Muhammad Zeeshan $^{4}$, Muhammad Zeshan Ahmad $^{5}$ \\ ${ }^{1}$ Riphah College of Rehabilitation Sciences, Riphah International University, Lahore Pakistan \\ ${ }^{2}$ Assistant Professor of Riphah College of Rehabilitation \& Allied Health Sciences, Riphah International University Lahore \\ ${ }^{3}$ Riphah Institute of Clinical and Professional Psychology, Riphah International University, Lahore Pakistan \\ ${ }^{4}$ Riphah College of Rehabilitation Sciences, Riphah International University, Lahore Pakistan \\ ${ }^{5}$ Lecturer at Department of Health Sciences, University of South Asia, Raiwind Road Lahore Campus
}

*Corresponding author: Fatima Mehmood; psychologistfatima15@gmail.com

Received: 12 October 2020;

Accepted: 28 October 2020;

Published: 01 November 2020

\begin{abstract}
Plantar Fasciitis is among the major causes of heel pain. Mechanical stress of plantar aponeurosis usually causes inflammation thus result in problem with weight bearing and difficulty in walking. Pain often exacerbates and may stick at for months with unremitting activity, limiting the activities of daily living and the functional status. The objective of the study was to compare the effects of low level laser and ultrasound therapy in patients for pain and their functional status presents with plantar fasciitis. This study was a Quasi-experimental trial and conducted at Physical therapy Center for Arthritis \& Rehabilitation Excellence (PT CARE), Gulberg III Lahore. The study completed in the time duration of six months (Aug 2019 - Jan 2020). Consecutive sampling technique was used to collect the data. A sample size of 'total 28 patients were taken in this study; patients were divided into two groups. Conventional Physiotherapy protocol was given to both groups as baseline treatment. (Group A patients were treated with low level laser protocol whereas Group B was treated with ultrasound therapy protocol). Functional Foot Index (FFI) score was used to ask some questions related to patients symptoms and daily activities and to measure the intensity of pain Numeric Pain Rating Scale (NPRS) was used. All participants of the study filled the FFI \& NPRS score and Numeric pain rating scale on day 1 in first cycle as pretreatment values and at the end of 5th session and 10th session as first and second cycles as post treatment values respectively. Data was analyzed on SPSS 21.The means of pre-treatment NPRS in group A was 7.78 and in group B means of pre-treatment NPRS was 7.35 with p value $<0.05$. Means difference of NPRS for group A was 4.07 and in group B the post treatment- 1 means difference 4.85 with $p$ value $<0.05$. Means of post treatment-2 in group A was 1.71 and in group B was 2.64 with p value $<0.05$. While means of pre-treatment in group A for FFI was 77.66 in group A and group B was 73.35 with p value $<0.05$. Means of post treatment-1 for group A for FFI was 54.50 and for group B 58.27 with $\mathrm{p}$ value $<0.05$. And means of post treatment- 2 in FFI for group-A was 28.22 and for group B was 35.88 with $p$ values $<0.05$. Low Level Laser Therapy is more effective than Ultrasound Therapy in reducing pain and improving functions in patients with plantar fasciitis and vice versa.
\end{abstract}

\section{Introduction}

Heel pain as a predominant cause referred to planter fasciitis. The occurrence of Planter Fasciitis is projected at $10 \%$ in general population, and presentation is common in 40 to 60 years old age group individuals especially including obese individual, women, athlete and soldiers.

\section{Objective}

As a common disease the Plantar fasciitis Regrettably the conventional therapies does not show effectiveness to all patients and to be a pain free after the PF have less chances and there are many patients which in search of relief having the chronic pain due to it. Stepwise progressive therapies are used to resolve the symptoms with most common sequence of steroidal injection, shock wave therapy, injection of plasma rich in platelets in human tissue, cryo-therapy with physical therapy and at the end surgery either micro-debridement or fasciotomy. Possibly, if there the presence of supplementary therapies were present to reduce the pain, and the expedition of advanced and more aggressive remedies could be halted.

Now therapies with different forms of energy are available to treat the plantar fasciitis and all these form showed the satisfactory results. These include the ultrasound therapy, ultrasound-guided pulsed radiofrequency (UG-PRF) treatment which is newly established for the gastrocnemius, noninvasive interactive neuro-stimulation (NIN) low-level laser therapy (LLLT), radiotherapy (i.e., radiation therapy), and intra-corporeal pneumatic shock therapy (IPST). These interventions uses different forms of energy for treatment purpose are the sonic wave for mechanical fluctuations, heat and light which propagate through a 
medium in distinction to surgical approaches or injection therapy in planter fasciitis patients. Stimulus frequency, intensity, pulse interval, treatment duration, and technique of application are the main factor which effects the effectiveness of these therapies (e.g., minimally invasive).

\section{Approach}

Planter fasciitis treatment portion of physical therapy which based on therapeutic exercises and electric modalities include ultrasound method in which therapeutic benefits of heat assumed to be delivered within the body. Ultrasound (US) was considered electrical energy is not; instead it is a mechanical form of energy, it is the therapeutic effect of mechanical energy for which it has been used for tissue healing. It is proposed that the solicitation of Ultrasound to the injured tissues speed up the rate of healing and augment the quality of the healing. It's from 1930 that the ultrasound of various types have been used for the soft tissue injuries treatment. Low energy, pulses of long duration and diffuse form are the most commonly used form of conventional therapeutic ultrasound which warm the soft tissue under the ultrasonic beam. ITU is the form which produce the concentrated sound waves to generate the selective thermal congeal effect so that the small area of required tissue should be involved and the remaining portion of tissue remain unaffected, it's the newly developed ultrasound centered therapy. Other than planter fascia ITU is approved from U.S. FDA and the CE mark in the market for the use of facial skin treatment and submental tissue lifting with non-surgical brow clinically from decades. From this technology more than 3 million patients receive the treatment and benefited worldwide. About $85 \%$ of the subjects receiving ITU treatment showed the improvement for facial skin treatment in which facial lifting, inflammation, pain, scaring and erythema are the main characters of measurements for condition improvement. Dermal collagen production, elastic fiber straightening and the dermis thickening are the main histological induction done by ITU type of treatment.

Laser therapy (LLLT) with low intensity is another noninvasive method of treatment for planter fasciitis. It's a painless and noninvasive method for the treatment of planter fasciitis patients. Laser therapy (LLLT) with low energy is used with assumption that wound healing with pain reduction and inflammation alleviation can be accelerated with it. On molecular level photobiomodulation and the photobiostimulation are the two main phenomena which help in tissue regeneration and the cell proliferation are the two main function of low energy level laser therapy. Principles of photochemistry dictate the Laser therapy with low intensity (LLLT) via photochemical or non-thermal effects on cells. Pain relieved by LLLT, a treatment protocol for PF become common in current years. Total score of American Orthopedic Foot and Ankle Society Score showed significant improvement in function after using LLLT for 3 weeks which in turn improve the walking even over uneven surface and also increase in walking distance reported by cinar et al. planter fascia show improvement in its thickness which decreased to a significant level interventional group as compared to control group monitor by ultrasound guided imaging.

\section{Organization of this Thesis}

The existence of evidence supports the use of conservative treatment options such as LASER with low intensity and ultrasonic therapy in patients of plantar fasciitis. It has been proposed that pain and inflammation are primary complaints in plantar fasciitis and decreasing inflammation is associated with increase functional capability of patients. There is limited data available comparing the effects of laser with low intensity and ultrasonic therapy in patients of plantar fasciitis. There is prerequisite to compare the effects of laser with low intensity and ultrasound therapy. This study envisioned to define the clinical effects of low level laser and ultrasound therapy.

\section{Literature Review}

Shrestha B et al done a prospective studyin 2018 at westeran Nepal to compare the ultersound and corticosteroid injection in patients of planter fascitis with sample size of 60 patients they conclude that both Ultrasound therapy with stretching exercise and steroid injection with stretching exercise were actual methods for moderating pain and refining function of the foot in plantar fasciitis. It was seen that steroid injection was more superior to the Ultrasound therapy combined with exercise. Micheal et al conducted a comparative study which revealed the efficiency of ITU in patients suffering of chronic planter fasciitis for pain relief. Moreover enhancement of daily living activities, reduction in size of hypoechoic lesion, level of satisfaction improvement of patients reported by them-selves provides the surplus evidence in the effectiveness of ITU as one of noninvasive. It's a short duration treatment with the follow-up of 6 month showing effectiveness in reduction of pain as compared to control group.

Evelyn hiegh et al in 2018 conducted a study for planter fasciitis of chronic in nature for the effectiveness of therapeutic ultrasound at intensive level to evaluate the musculoskeletal tissue pain lessening in a fundamental clinical trial to examine the tolerance of patients, efficacy and wellbeing. In this singly blinded study, 2 treatments were provided to 33 patients and both were apart 4 weeks from 1 st session to next along with conventional protocol which established for the plantar fascia. Six months follow up were followed after the first session of treatment of patient, in which he had been examine physically and through ultrasound of diagnostic category at each follow-up visit and completing patient/subject-reported outcome measure and Foot Function Index surveys. About $\geq 25 \%$ reduction in pain both on individuality and average. Hypo echoic area variations and adversarial proceedings were restrained on diagnostic ultrasound. Scoring of the pain during the treatment 1 was with mean of 3.4 , and for 2 nd treatment it was mean of 2.9. There was an Attrition of a single patient due to occurrence of pain, after the 1 st session. Other than that no hostile actions were noted. In this essential clinical trial of chronic planter fasciitis the intense therapeutic ultrasound has shown a good effectiveness, safety and tolerance during this study.

\section{Hypothesis}

Alternate hypothesis: Low Level Laser Therapy is more effective than Ultrasound Therapy in reducing pain and improving functions in patients with plantar fasciitis and vice versa.

Null hypothesis: Low Level Laser Therapy and Ultrasound Therapy are equally effective in reducing pain and improving functions in patients with plantar fasciitis.

\section{Method}

\section{Study Design}

This study was a Quasi-experimental trial. 


\section{Sample Size}

Sample size was 26, which calculated by online EPITOOL sample size calculator by putting following values of NPRS from previous study:

Population mean in Group 1

$$
=2.9
$$

Variance in Group I

$$
=1.18
$$

Population mean in Group II $\quad=1.69$

Variance in Group II $\quad=1.1$

Confidence Level $\quad=0.95$

Power $\quad=0.80$

Margin of Error $\quad=5 \%$

Ratio of sample sizes (n2/n1) $\quad=1$

Tailed Test $\quad=2$

Group $1(\mathrm{n} 1) \quad=13$

Group II (n2) = $\quad=13$

Total Sample size $\quad=26$

After addition of $10 \%$ Attrition rate a sample size of 28 is calculated.

\section{Study Groups}

Group A: Low Level Laser Therapy. \& stretching exercises Group B: Ultrasound Therapy \& stretching exercises.

\section{Sampling Technique}

Consecutive sampling technique was used to collect the data.

\section{Sample Selection}

The tenderness which was localized on the calcaneal medial tubercle and the morning pain which began at the first step and show the worsening after the weight bearing activities are the main bases of planter fasciitis diagnosis.

\section{Inclusion Criteria}

Inclusion criteria was based on recommendation in Clinical Practice Guidelines for Heel pain \& Plantar Fasciitis (Revision 2014), which is linked on health form disability and functioning to the American physical therapy association International Classification section of orthopedic.

- Heel pain upon the proximal insertion of planter fascia palpation produced tenderness.

- Heel Pain which is none radiating in nature, sharp and localized.

- Heel Pain triggered by a latest increase in weight-bearing activity

- $\quad$ Pain reduced primarily after taking the first steps but worsened as there increased in activity

- Movement at the talocrural joint especially dorsiflexion, active and passive is limited.

\section{Exclusion Criteria}

- Patients with steroid injection history.

- History of foot surgery, herniation of lumber disc or any other injury of back.

- Peripheral joint stiffness, redness, swelling, warmth deformities and the erythema like skin changes, patients with these characteristics were excluded from the study.

\section{Data Collection Tool}

1. Functional Foot Index (FFI)

2. Numeric pain rating scale (NPRS)

\begin{tabular}{|c|c|c|c|c|c|}
\hline \multicolumn{2}{|c|}{ Treatment groups of patients } & $\mathbf{N}$ & Minimum & Maximum & Mean \pm SD. \\
\hline \multirow{4}{*}{ Low Level Laser Therapy } & Height & 14 & 154 & 174 & $165+006$ \\
\hline & Heignt & 14 & 1.54 & 1.14 & $1.05 \pm 0.00$ \\
\hline & Weight & 14 & 57.00 & 87.00 & $72.35 \pm 9.62$ \\
\hline & BMI & 14 & 21.99 & 29.94 & $26.96 \pm 2.44$ \\
\hline \multirow{3}{*}{ Ultrasound Therapy } & Height & 14 & 1.51 & 1.77 & $1.63 \pm .070$ \\
\hline & Weight & 14 & 51.00 & 94.00 & $72.78 \pm 13.14$ \\
\hline & BMI & 14 & 18.96 & 32.47 & $26.96 \pm 3.64$ \\
\hline
\end{tabular}

Table 1: Age, Weight, Height and BMI of Patients

Table 1 shows the basic demographics of the study such as weight, height and BMI of the patients. Mean Weight of patients was $72.78+13.14$, with maximum weight $94 \mathrm{~kg}$ and minimum weight $56 \mathrm{~kg}$.in Experimental group. Mean Weight of patients was $70.80+6$. with maximum weight $51 \mathrm{~kg}$.and minimum weight $57 \mathrm{~kg}$. Mean weight of patients was $72.35+9.62$, with maximum weight 87 and minimum weight $57.00 \mathrm{~kg}$. Mean Height of patients was $1.63+.070$, with maximum Height 1.77 meters and minimum Height 1.51 meters in Experimental group. Mean Height of patients was $1.65+0.06$, with maximum Height 1.74 meters .and minimum Height 1.54 meters. In Control group. Similarly Mean BMI of patients was $26.96+3.64$, with maximum BMI 32.47 and minimum BMI 18.96 in Experimental group. Mean BMI of patients was $26.96+2.44$, with maximum BMI 29.94 and minimum BMI 21.99 in Control group.

Table 2: Gender of the Patients

\begin{tabular}{|l|l|l|l|}
\hline \multicolumn{2}{|l|}{ Gender of the patients } & Frequency & Percent \\
\hline \multicolumn{2}{|l|}{ Treatment groups of patients } & 6 & 42.8 \\
\hline \multirow{2}{*}{ Low Level Laser Therapy } & Male & 8 & 57.1 \\
\cline { 2 - 4 } & Female & 4 & 28.5 \\
\hline \multirow{2}{*}{ Ultrasound Therapy } & Male & 10 & 71.42 \\
\cline { 2 - 4 } & Female & 10 & \\
\hline
\end{tabular}

Table 2 shows that $42.8 \%(n=6)$ males and $57.1 \%(n=8)$ females were in control group. While $32.1 \%(n=4)$ males and $67.8 \%$ ( $=10)$ females were in Experimental group. 
Table 3: Duration of Pain

\begin{tabular}{|l|l|l|l|}
\hline Group & & Frequency & Percent \\
\hline \multirow{4}{*}{ Low Level Laser Therapy } & All the time & 7 & 50.0 \\
\cline { 2 - 4 } & Pain when standing and walking & 4 & 28.6 \\
\cline { 2 - 4 } & Pain starts after long standing or weight bearing & 3 & 21.4 \\
\hline \multirow{3}{*}{ Ultrasound Therapy } & All the time & 3 & 21.4 \\
\cline { 2 - 4 } & Pain when standing and walking & 7 & 50.0 \\
\cline { 2 - 4 } & Pain starts after long standing or weight bearing & 4 & 28.6 \\
\hline
\end{tabular}

Table 3 shows Duration of pain in experimental group in which patients having pain all the time 50\% ( $\mathrm{n}=7$ ), patients with pain standing and walking $28 \%(\mathrm{n}=4)$ and patients with after long standing or weight bearing $21 \%(\mathrm{n}=3)$.

Duration of pain in controlled group in which patients having pain all the time $21 \%(\mathrm{n}=3)$, patients with pain standing and walking $50 \%(\mathrm{n}=7)$ and patients with after long standing or weight bearing $28 \%(n=4)$.

Table 4: Pair wise within group comparison of NPRS (Repeated measure ANOVA)

\begin{tabular}{|c|c|c|c|c|}
\hline & \multicolumn{2}{|c|}{ Group A mean } & \multicolumn{2}{|c|}{ Group B mean } \\
\hline Pre treatment NPRS & \multicolumn{2}{|l|}{7.78} & \multicolumn{2}{|l|}{7.35} \\
\hline Post-treatment I NPRS & \multicolumn{2}{|l|}{4.07} & \multicolumn{2}{|l|}{4.85} \\
\hline \multirow[t]{2}{*}{ Post treatment II NPRS } & \multicolumn{2}{|l|}{1.71} & \multicolumn{2}{|l|}{2.64} \\
\hline & Mean Diff & P value & Mean Diff & P value \\
\hline Pre treatment NPRS - Post treatment I NPRS & 3.71 & $<0.05$ & 2.500 & $<0.05$ \\
\hline Post treatment I NPRS- Post treatment II NPRS & 2.35 & $<0.05$ & 2.21 & $<0.05$ \\
\hline Pre treatment NPRS - Post treatment II NPRS & 6.07 & $<0.05$ & 4.71 & $<0.05$ \\
\hline
\end{tabular}

The results shows that in Group A mean value of pain intensity on numeric pain rating scale (NPRS) at pre-treatment was $7.78+0.214$ Posttreatment I NPRS $4.07+0.221$ after post treatment I which was further reduced to $1.71+0.163$ post treatment II in Experimental group. The results shows that mean value of pain intensity on numeric pain rating scale (NPRS) in GROUP B pre-treatment NPRS was $7.35+.269$ reduced to $4.85+0.20$ after post treatment I NPRS reduced to $2.64+.225$ after post treatment II NPRS which was less evident than experimental group.

In Group A Mean (I-J) diff .between pretreatment NPRS -post treatment I NPRS was 3.71 with p value <0.05, Mean I-J difference between post treatment 1 NPRS -post treatment II NPRS was 2.35 with value $<0.05$ and mean I-J difference between Post treatment II NPRS and pretreatment NPRS was 6.07 with $\mathrm{p}$ value $<0.05$.

In Group B Mean (I-J) diff .between pretreatment NPRS -post treatment I NPRS was 2.50 with p value $<0.05$, Mean I-J difference between post treatment 1 NPRS -post treatment II NPRS was 2.21 with value $<0.05$ and mean I-J difference between Post treatment II NPRS and pretreatment NPRS was 4.71 with $p$ value $<0.05$.In both groups significant improvement in NPRS was found.

Table 5: pair wise across the group comparison of NPRS (Mixed model ANOVA)

\begin{tabular}{|l|l|l|}
\hline \multicolumn{2}{|l|}{} \\
\hline Pretreatment NPRS & Mean \\
\hline Post treatment I NPRS & 7.57 & \multicolumn{2}{l|}{} \\
\hline Post treatment II NPRS & 4.46 & Palue \\
\hline & 2.17 & $<0.05$ \\
\hline Pretreatment NPRS - Post treatment I NPRS & Mean Difference & $<0.05$ \\
\hline Post treatment I NPRS - Post treatment II NPRS & 3.10 & $<0.05$ \\
\hline Pretreatment NPRS - Post treatment II NPRS & 2.28 & 5.39 \\
\hline
\end{tabular}

Table 5 shows across the group comparison of NPRS at pretreatment 7.57, 4.46 after post treatment I and 2.17 after post treatment II. The mean (I-J) difference between pretreatment NPRS and post treatment I NPRS across the group was 3.10 with p value $<0.05$, mean (I-J) difference between post treatment I NPRS and Post treatment II NPRS was 2.28 with $\mathrm{p}$ value $<0.05$ and mean (I-J) difference between post treatment II and pretreatment NPRS was 5.39 with $\mathrm{p}$ value $<0.05$ showing that there is significant difference in NPRS improvement across the group.

Table 6: pair wise within group comparison of FFI (Repeated measure ANOVA)

\begin{tabular}{|c|c|c|c|c|}
\hline & \multicolumn{2}{|l|}{ Group A mean } & \multicolumn{2}{|l|}{ Group B mean } \\
\hline Pre-treatment FFI & \multicolumn{2}{|l|}{77.66} & \multicolumn{2}{|l|}{73.35} \\
\hline Post treatment I FFI & \multicolumn{2}{|l|}{54.50} & \multicolumn{2}{|l|}{58.27} \\
\hline \multirow[t]{2}{*}{ Post treatment II FFI. } & \multicolumn{2}{|l|}{28.22} & \multicolumn{2}{|l|}{35.88} \\
\hline & Mean Difference & $P$ value & Mean Difference & P value \\
\hline Pretreatment FFI - Post treatment I FFI & 23.16 & $<0.05$ & 28.27 & $<0.05$ \\
\hline Post treatment I FFI- Post treatment II FF1 & 23.16 & $<0.05$ & 28.27 & $<0.05$ \\
\hline Pre-treatment FFI - Post treatment II FFI & 51.44 & $<0.05$ & 51.44 & $<0.05$ \\
\hline
\end{tabular}

The results shows that in Group A mean value OF functional foot index (FFI) pre-treatment was $77.66+.978$ reduced to $54.50+1.61$ after post treatment I which was further reduced to $28.22+0.978$ after post treatment II FFI.The results shows that mean value of pain intensity on 
functional foot index (FFI) in group B pre-treatment was $73.35+1.17$ reduced to $58.27+1.61$ after post treatment I reduced to $35.88+1.17$ after post treatment II which was less evident than experimental group.

Table 7: Pair wise across the group comparison of FFI (Mixed model ANOVA)

\begin{tabular}{|l|l|l|}
\hline \multicolumn{2}{|l|}{} & Mean \\
\hline Pre-treatment FF1 & 75.51 \\
\hline Post treatment I FF1 & 56.38 \\
\hline Post treatment II FF1 & 31.05 & P value \\
\hline & Mean Difference & $<0.05$ \\
\hline Pre -treatment FF1 - Post treatment I NPRS & 19.12 & $<0.05$ \\
\hline Post treatment I FF1 at 1st session - Post treatment II FF1 & 25.33 & $<0.05$ \\
\hline Pre -treatment FF1 - Post treatment II NPRS & 44.45 & \\
\hline
\end{tabular}

Table no.7 across the group comparison of FFI at pretreatment 75.51, 56.38 after post treatment I and 31.05 after post treatment II.The mean (IJ) difference between pretreatment FFI and post treatment I FFI across the group was 19.12 with p value $<0.05$, mean (I-J) difference between post treatment I FFI and Post treatment II FFI was 25.33 with p value $<0.05$ and mean (I-J) difference between post treatment II and pretreatment FFI was 44.45 with $\mathrm{p}$ value $<0.05$ showing that there is significant difference in FFI improvement across the group.

\section{Discussion}

In a recent study done by raja Muhammad kamel et al. in Cairo University Egypt using low level laser therapy verses ultrasound shows that group receiving low level laser therapy show better results as compared to ultrasound group and control group. Which support the hypothesis current study about the low level laser efficacy in comparison to ultra-sound. In this research all the variables were investigated and proposed in the light of the previous indigenous and international researches. It is determined that low intensity laser is more beneficial than ultrasound in plantar fasciitis patients.

Ordahan (2018) conducted research on the effects of laser with high-intensity in comparison to laser of low-level managing the plantar fasciitis by using visual analog score and foot and ankle functional index with results showing that there were comparable results either by HILT or by LLLT group verified healthier progress in all parameters against each other. Both engagements emend the pain levels, along with the quality of life and functional level of PF patients. A systematic review conducted by Wang (2018) with meta-analysis done to appraise the effect of treatment of LLLT in PF patients. Inclusive suggestion of the analysis was that the LLLT can considerably release pain for three month of PF after treatment. Another research conducted by cinar (2018), using low laser against the extra corporeal shock wave protocol for the planter fasciitis treatment with functional foot index and numeric rating of pain scale and his research revealed that the therapy of LLLT as compared to ESWT shows more proficient results in improvement of pain and functional index of foot although both cause sufficient improvement in overall.

After the interpretation of previous studies with the results of current study it favored the results and hypothesis of current study that low level of laser therapy in comparison to ultrasound. But it's not an absoluteness to some way of treatment for the patients of planter fasciitis to strict upon the use of low level laser therapy as there are many factors present to effect any of the research in its results so also they do in present study.

\section{Conclusion}

Low Level Laser Therapy is more effective than Ultrasound Therapy in decreasing pain and refining functions in plantar fasciitis patients and vice versa.

\section{Conflicts of Interest}

"The author declare that there is no conflict of interest regarding the publication of this paper."

\section{References}

[1] Beeson P. Plantar fasciopathy: revisiting the risk factors. Foot and Ankle Surgery. 2014;20(3):160-5.

[2] Goweda RA, Alfalogy EH, Filfilan RN, Hariri GA. Prevalence and Risk factors of Plantar Fasciitis among Patients with Heel Pain Attending Primary Health Care Centers of Makkah, Kingdom of Saudi Arabia. Journal of High Institute of Public Health. 2015;45(2):71-5.

[3] Tahririan MA, Motififard M, Tahmasebi MN, Siavashi B. Plantar fasciitis. Journal of research in medical sciences: the official journal of Isfahan University of Medical Sciences. 2012;17(8):799.

[4] Cleland JA, Abbott JH, Kidd MO, Stockwell S, Cheney $\mathrm{S}$, Gerrard DF, et al. Manual physical therapy and exercise versus electrophysical agents and exercise in the management of plantar heel pain: a multicenter randomized clinical trial. journal of orthopaedic \& sports physical therapy. 2009;39(8):573-85.

[5] Neufeld SK, Cerrato R. Plantar fasciitis: evaluation and treatment. JAAOS-Journal of the American Academy of Orthopaedic Surgeons. 2008;16(6):338-46.

[6] Van Leeuwen K, Rogers J, Winzenberg T, van Middelkoop M. Higher body mass index is associated with plantar fasciopathy/'plantar fasciitis': systematic review and meta-analysis of various clinical and imaging risk factors. Br J Sports Med. 2016;50(16):972-81.

[7] Salvioli S, Guidi M, Marcotulli G. The effectiveness of conservative, non-pharmacological treatment, of plantar heel pain: A systematic review with meta-analysis. The Foot. 2017;33:57-67.

[8] Riddle DL, Schappert SM. Volume of ambulatory care visits and patterns of care for patients diagnosed with plantar fasciitis: a national study of medical doctors. Foot $\&$ ankle international. 2004;25(5):303-10.

[9] Heigh E, Bohman L, Briskin G, Slayton M, Amodei R, Compton K, et al. Intense Therapeutic Ultrasound for Treatment of Chronic Plantar Fasciitis: A Pivotal Study Exploring Efficacy, Safety, and Patient Tolerance. The Journal of Foot and Ankle Surgery. 2019;58(3):519-27.

[10] Martin RL, Davenport TE, Reischl SF, McPoil TG, Matheson JW, Wukich DK, et al. Heel pain-plantar 
fasciitis: revision 2014. Journal of Orthopaedic \& Sports Physical Therapy. 2014;44(11):A1-A33.

[11] Lim AT, How $\mathrm{CH}$, Tan B. Management of plantar fasciitis in the outpatient setting. Singapore medical journal. 2016;57(4):168

[12] Miller DL, Smith NB, Bailey MR, Czarnota GJ, Hynynen K, Makin IRS, et al. Overview of therapeutic ultrasound applications and safety considerations. Journal of Ultrasound in Medicine. 2012;31(4):623-34.

[13] Gliklich RE, White WM, Slayton MH, Barthe PG, Makin IRS. Clinical pilot study of intense ultrasound therapy to deep dermal facial skin and subcutaneous tissues. Archives of facial plastic surgery. 2007;9(2):88-95.

[14] Suh DH, Shin MK, Lee SJ, Rho JH, Lee MH, Kim NI, et al. Intense focused ultrasound tightening in Asian skin: clinical and pathologic results. Dermatologic surgery. 2011;37(11):1595-602.

[15] White WM, Makin IRS, Barthe PG, Slayton MH, Gliklich RE. Selective creation of thermal injury zones in the superficial musculoaponeurotic system using intense ultrasound therapy: a new target for noninvasive facial rejuvenation. Archives of facial plastic surgery. 2007;9(1):22-9.

[16] Laubach HJ, Makin IR, Barthe PG, Slayton MH, Manstein D. Intense focused ultrasound: evaluation of a new treatment modality for precise microcoagulation within the skin. Dermatologic surgery. 2008;34(5):72734.

[17] Cinar E, Saxena S, Uygur F. Low-level laser therapy in the management of plantar fasciitis: a randomized controlled trial. Lasers in medical science. 2018;33(5):949-58.

[18] Wang W, Jiang W, Tang C, Zhang X, Xiang J. Clinical efficacy of low-level laser therapy in plantar fasciitis: A systematic review and meta-analysis. Medicine. 2019;98(3).

[19] Cinar E, Saxena S, Uygur F. Combination therapy versus exercise and orthotic support in the management of pain in plantar fasciitis: a randomized controlled trial. Foot \& ankle international. 2018;39(4):406-14.

[20] Shrestha B, Sapkota K. Effectiveness of ultrasound therapy versus steroid injection in plantar fasciitis patients in western region of Nepal. International Journal of Orthopaedics. 2018;4(3):255-8.

[21] Slayton MH, Baravarian B, Amodei RC, Compton KB, Christensen DN, McNelly A, et al. Intense Therapeutic Ultrasound for Pain Relief in the Treatment for Chronic Plantar Fasciopathy. Foot \& Ankle Orthopaedics. 2019;4(3):2473011419862228.

[22] Ulusoy A, Cerrahoglu L, Orguc S. Magnetic resonance imaging and clinical outcomes of laser therapy, ultrasound therapy, and extracorporeal shock wave therapy for treatment of plantar fasciitis: a randomized controlled trial. The Journal of Foot and Ankle Surgery. 2017;56(4):762-7.

[23] Mustafa MS, Saleh MMS. Low level laser versus ultrasound on myofascial trigger points of planter fasciitis. International Journal of Therapies and Rehabilitation Research. 2017;6(5):25-32.

[24] Ordahan B, Karahan AY, Kaydok E. The effect of highintensity versus low-level laser therapy in the management of plantar fasciitis: a randomized clinical trial. Lasers in medical science. 2018;33(6):1363-9.

[25] Kiritsi O, Tsitas K, Malliaropoulos N, Mikroulis G. Ultrasonographic evaluation of plantar fasciitis after lowlevel laser therapy: results of a double-blind, randomized, placebo-controlled trial. Lasers in medical science. 2010;25(2):275-81.

[26] Macias DM, Coughlin MJ, Zang K, Stevens FR, Jastifer JR, Doty JF. Low-level laser therapy at $635 \mathrm{~nm}$ for treatment of chronic plantar fasciitis: a placebocontrolled, randomized study. The Journal of Foot and Ankle Surgery. 2015;54(5):768-72.

[27] Agel J, Beskin JL, Brage M, Guyton GP, Kadel NJ, Saltzman CL, et al. Reliability of the Foot Function Index: a report of the AOFAS Outcomes Committee. Foot \& ankle international. 2005;26(11):962-7. 